\title{
Seroprevalence and Associated Factors of Toxoplasma gondii Infection among Pregnant Women Attending in Antenatal Clinic of Arba Minch Hospital, South Ethiopia: Cross Sectional Study
}

\author{
Tsegaye Yohanes ${ }^{1}$, Zerihun Zerdo ${ }^{1}$, Nega Chufamo ${ }^{2}$ and Ashenafi Abossie ${ }^{1}$ \\ ${ }^{1}$ Department of Medical Laboratory Science, Arba Minch University, Arba Minch, Ethiopia \\ ${ }^{2}$ Department of Obstetrics and Gynecology, School of Medicine, Arba Minch University, Arba Minch, Ethiopia
}

Corresponding author: Tsegaye Yohanes, Department of Medical Laboratory Science, Arba Minch University, Ethopia, Tel: +251919904201; Email: tsegaye.yohanes@yahoo.com

Received Date: February 01, 2017; Accepted Date: February 15, 2017; Published Date: February 20, 2017

Citation: Yohanes T, Zerdo Z, Chufamo N, et al. Toxoplasma gondii Infection: Seroprevalence and associated Factors among Pregnant Women Attending in Antenatal Clinic of Arba Minch Hospital, South Ethiopia: Cross Sectional Study. Transl Biomed. 2017, 8:1.

\section{Abstract}

Background: Toxoplasma gondii (T. gondii) is a ubiquitous, coccidian intracellular protozoan parasite that causes toxoplasmosis. T. gondii infection acquired during pregnancy may result in severe damage or death of the fetus and long-term sequelae in offspring. So far, no documented data concerning the seroprevalence of $T$. gondii infection among pregnant women is available in the study area. Therefore, this study was aimed at determining of $T$. gondii sero-prevalence and associated factors among pregnant women attending in ante natal clinic (ANC) of Arba Minch hospital, southern Ethiopia.

Methods: A facility based cross sectional study design was employed. A total of 232 pregnant women visiting Arba Minch hospital ANC from February to April, 2015 were enrolled by using systematic sampling technique. Data regarding Socio-demographic and associated factors were gathered using pretest questionnaire. Approximately 2 milliliters of blood specimen was collected and serum samples were tested for anti-T. gondii IgG and IgM antibody using Enzyme Linked Immunosorbent Assay. Data were analyzed using SPSS version 20.

Results: Out of the total 232 pregnant women tested, 184 were found to be seropositive, giving overall seroprevalence rate of $T$. gondii infection $79.3 \%(95 \% \mathrm{Cl}$; 73.7-84.5). On multivariate analysis showed that consumption of raw meat (AOR=3.211; 95\% Cl: 1.592-6.477) and habit of eating raw vegetables or fruit (AOR=2.669; 95\% Cl: 1.264-5.639) were significantly associated with $T$. gondi infection.

Conclusion: The overall seroprevalence of $T$. gondii infection among pregnant women was high and serological evidence of primary infection was observed. Therefore, screening of Toxoplasma infection should be considered during ANC follow up. Moreover, health information about ways to minimize exposure to the risk factors should be provided with main focus on not eats raw meat and raw vegetables.

Keywords: Seroprevalence; T. gondii; Pregnant women; Ethiopia

\section{Introduction}

Toxoplasma gondii ( $T$. gondii) is a causative agent of Toxoplasmosis disease; it is an intercellular parasite infected humans and other warm blooded animals. Globally approximately a half of the world populations are infected $[1,2]$. Bradyzoites, tachyzoites and the sporozoites in oocysts are the three infectious stages of $T$. gondii parasite to all hosts including human being [3]. Humans usually acquire T. gondii infection through consumption of raw or undercooked meat. It is also contracted through the consumption of improperly washed vegetables and fruits as well as drinking of water which contains oocysts. Moreover, trans placentally tachyzoites infect fetus in pregnant women [4,5].

Toxoplasmosis is normally asymptomatic in healthy individuals but can cause maternal-fetal transmission in women who acquire primary toxoplasma infection during pregnancy $[6,7]$. The risk of vertical transmission and associated problems are determined by the gestational age at which the primary infection is acquired. Transmission to the fetus increases from the first trimester (10\% to $24 \%$ ) to the third trimester (60\% to $90 \%)$, but the potential of congenital defect is more severe with earlier infections $[8,9]$.

Diagnosis of toxoplasmosis in humans is performed using different techniques. Acute and latent $T$. gondii infections during pregnancy are mostly diagnosed by serological tests including detection of anti-T. gondii-specific IgM and IgG antibodies [10]. Latex agglutination (LA) testing, enzyme-linked immunosorbent assay (ELISA), and/or indirect fluorescent antibody testing (IFAT) are some of the tests that used to detect the antibodies [11]. 
Prevalence of $T$. gondii infection in pregnant women varies greatly among different countries. Seroprevalence of $10.3 \%$ and $69 \%$ reported from Japan [12] and Northeastern Brazil [13]. In Africa, data on T. gondii infection during pregnancy is scant and also the burden of $T$. gondii infection in pregnant women is an under-estimated public health concern. In these countries, higher up to $92.5 \%$ seroprevalence has been reported. Pregnant women are not routinely investigated for $T$. gondii during pregnancy, and follow-up does not exist [14,15]. In Ethiopia, toxoplasmosis is a cause of multiple diseases in humans [16]. Seroprevalence of $83.6 \%, 81.4 \%$ and $88.2 \%$ were also reported among pregnant women, in women of childbearing age and HIV infected individuals from Jimma [17] Central Ethiopia [18] and Arba Minch [19] respectively.

Prevalence of $T$. gondii infection and the relative contribution of the various routes of transmission in humans have not been adequately studied In Ethiopia [20]. There is also little information concerning seroprevalence of Toxoplasma infection in general and in pregnant women particularly. Moreover, laboratory diagnosis of Toxoplasma infection is currently not practiced in all health facilities of the country. To our knowledge, the seroprevalence of $T$. gondii infection in pregnant women particularly have not previously been reported in the study area. Therefore, the present study was aimed to determine sero-prevalence of $T$. gondii infection and to identify associated risk factors among pregnant women attending ANC in Arba Minch hospital, South Ethiopia.

\section{Methods}

\section{Study design and setting}

A facility based cross sectional study was conducted in Arba Minch Hospital ANC clinics. Arba Minch hospital is found in Arba Minch Town located 505 kilometers in the southern part from Addis Ababa, capital city of Ethiopia and serves more than 2 million people in South Nations Nationalities and Peoples Regional State in Ethiopia. The hospital provides services such as Out Patient Department, Inpatients (medical, surgical, obstetrics and gynecology), ophthalmology, $\mathrm{MCH}$, pharmacy, laboratory and ART and TB clinics. The study was conducted from February to April, 2015.

\section{Sample size and sampling technique}

Sample size was determined using single population proportion formula to estimate the prevalence of $T$. gondii and $\mathrm{HBV}$ infection among pregnant mothers attending ANC in Arba Minch general hospital (large project). The sample size is calculated based on the following assumptions: prevalence of T. gondii infection among pregnant women $83.6 \%$ in Jimma [17] and prevalence of HBV in Mekelle (8.1\%) [21]; 95\% level of confidence, $5 \%$ margin of error and $10 \%$ non-response. Considering all the above constants the calculated sample size for T. gondii infection and HBV was 232 and 126. Finally, the larger sample size (232) was taken. Systematic sampling technique was used to recruit pregnant mothers in the study. A total of 696 pregnant women attended the ANC clinic during the past three months before study was initiated. This number was divided for the sample size to get the sample interval ( $k$ value) which is 3 . Therefore, every 3rd mother attending the clinic was enrolled in to the study until the calculated sample size was achieved within three months of data collection.

\section{Data collection}

A pre-tested questionnaire was used to collect information on socio-demographic and other predisposing factors to $T$. gondii infection. Nurses working in ANC clinic of the hospital was required, trained and collected the data by face-to-face interview. Following the interview, approximately $2 \mathrm{ml}$ of venous blood was collected aseptically from each consenting study participant. Then serum was separated and stored at $-20^{\circ} \mathrm{C}$ prior to assay. Finally, the serum was tested for anti- $T$. gondii IgM and IgG antibody using Enzyme Linked Immunosorbent Assay test kit (Human Gesellschaft für Biochemica und Diagnostica $\mathrm{mbH}$, Wiesbaden Germany) at Arba Minch National blood bank center laboratory, strictly following the manufacturer's instruction.

\section{Data analysis}

All collected data from questionnaire and laboratory were checked for completeness and consistency, and then the data was entered into computer, cleaned and analyzed using SPSS version 20.0 software package. Descriptive statistics was performed to describe demographic profile of the study participants. Bivariate and multivariate logistic regressions were used to assess the association between potential risk factors considered and $T$. gondii infection. Variables with $p$ value $<0.25$ by the bivariate analysis were entered into multivariate model. At multivariate logistic regression, $p$-value $<0.05$ was considered as statistically significant for all variables.

\section{Ethical considerations}

Before study is conducted, ethical clearance letter was obtained from Arba Minch University, College of Medicine and Health Science Research Ethical Review Committee. Permission to conduct the research was obtained from Arba Minch Zonal Health Bureau and Arba Minch Hospital. Moreover, written informed consent was obtained from all study participants prior to interview and blood collection. Confidentiality of the collected information and laboratory test results was maintained. Individual test results were communicated with the attending physician for further management of the cases.

\section{Results}

\section{Seroprevalence of T. gondii infection}

A total of 232 pregnant women of age between 15 to 38 years (mean 25.98 years) who attended Arba Minch Hospital ANC clinic were included and tested for anti-T. gondii IgG and IgM antibody, 184 were found to be seropositive, giving an 
overall prevalence of $79.3 \%(95 \% \mathrm{Cl} ; 73.7-84.5)$. Out of the total seropositive 175 (75.43\%) were only IgG seropositive, 9(3.9\%) were IgM seropositive and 2 of the 9 pregnant women were positive for both IgG and IgM.

In this study majority of pregnant women found within the age group 25-29 years which comprised 101 (43.5\%) of the total, out of which 79 (78.2\%) were seropositive. Two hundred fourteen $(92.2 \%)$ of the respondents were resided in urban area, of these 169 (79\%) were seropositive for T. gondii. Regarding to trimester 124 (53.4\%) pregnant women were found within second trimester, of which 98 (79\%) were seropositive for T. gondi (Table 1).

Table 1 Socio-demographic characteristics of pregnant women ( $n=232)$ attending Antenatal clinic at Arba Minch Hospital, 2015.

\begin{tabular}{|c|c|c|c|}
\hline \multirow[t]{2}{*}{ Variables } & \multicolumn{2}{|c|}{ Seroprevalence } & \multirow[t]{2}{*}{ Total No $(\%)$} \\
\hline & Positive n (\%) & Negative $\mathbf{n}(\%)$ & \\
\hline \multicolumn{4}{|l|}{ Age group (years) } \\
\hline $15-19$ & $12(75.0 \%)$ & $4(25.0 \%)$ & $16(6.9 \%)$ \\
\hline $20-24$ & $59(85.5 \%)$ & $10(14.5 \%)$ & $69(29.7 \%)$ \\
\hline $25-29$ & $79(78.2 \%)$ & $22(21.8 \%)$ & $101(43.5 \%)$ \\
\hline $30-34$ & $24(68.6 \%)$ & $11(31.4 \%)$ & $35(15.1 \%)$ \\
\hline $35-39$ & $10(90.9 \%)$ & $1(9.1 \%)$ & $11(4.7 \%)$ \\
\hline \multicolumn{4}{|l|}{ Residence } \\
\hline Urban & $169(79.0 \%)$ & $45(21.0 \%)$ & $214(92.2 \%)$ \\
\hline Rural & $15(83.3 \%)$ & $3(16.7 \%)$ & $18(7.8)$ \\
\hline \multicolumn{4}{|l|}{ Marital status } \\
\hline Married & $177(79.0 \%)$ & $47(21.0 \%)$ & $224(96.6 \%)$ \\
\hline Single & $5(83.3 \%)$ & $1(16.7 \%)$ & $6(2.6 \%)$ \\
\hline Divorced & $0(0.0 \%)$ & $0(0.0 \%)$ & $0(0.0 \%)$ \\
\hline Widowed & $2(100 \%)$ & $0(0.0 \%)$ & $2(0.9 \%)$ \\
\hline \multicolumn{4}{|l|}{ Educational status } \\
\hline Unable to read and write & $34(72.3 \%)$ & $13(27.7 \%)$ & $47(20.3 \%)$ \\
\hline Primary & $62(78.5 \%)$ & $17(21.5 \%)$ & $79(34.1 \%)$ \\
\hline Secondary & $59(83.1 \%)$ & $12(16.9 \%)$ & $71(30.6 \%)$ \\
\hline Tertiary & $29(82.9 \%)$ & $6(17.1 \%)$ & $35(15.1 \%)$ \\
\hline \multicolumn{4}{|l|}{ Occupation } \\
\hline Government & $52(77.6 \%)$ & $15(22.4 \%)$ & $67(28.9 \%)$ \\
\hline Housewife & $115(82.1 \%)$ & $25(17.9 \%)$ & $140(60.3 \%)$ \\
\hline Others * & $17(68 \%)$ & $8(32.0 \%)$ & $25(10.8 \%)$ \\
\hline \multicolumn{4}{|l|}{ Trimesters } \\
\hline First $(<14$ weeks $)$ & $32(82.1 \%)$ & $7(17.9 \%)$ & $39(16.8 \%)$ \\
\hline Second (14-28weeks) & $98(79.0 \%)$ & $26(21.0 \%)$ & $124(53.4 \%)$ \\
\hline Third (>28weeks) & $54(78.3 \%)$ & $15(21.7 \%)$ & $69(29.7 \%)$ \\
\hline \multicolumn{4}{|l|}{ Gravidity } \\
\hline Primigravidae & $67(78.8 \%)$ & $18(21.2 \%)$ & $85(36.6 \%)$ \\
\hline Multigravidae & $117(79.6 \%)$ & $30(20.4 \%)$ & $147(63.4 \%)$ \\
\hline
\end{tabular}


Among the total respondents, $153(65.9 \%)$ and $176(75.9 \%)$

respective prevalence of $T$. gondii infection among pregnant were reported to had the habit of eating raw meat and women was $86.9 \%(n=133)$ and $83.5 \%(n=147)$ (Table 2). unwashed/raw vegetables or fruits, respectively. The

Table 2 Bivariate and multivariate analyses of factors associated with T. gondii infection among pregnant women, Arba Minch Hospital, 2015.

\begin{tabular}{|c|c|c|c|c|}
\hline \multirow[t]{2}{*}{ Variables } & \multicolumn{2}{|l|}{ Sero-prevalence } & \multirow[t]{2}{*}{ COR $(95 \% \mathrm{Cl})$} & \multirow[t]{2}{*}{ AOR $(95 \% \mathrm{Cl})$} \\
\hline & Positive n (\%) & Negative $n(\%)$ & & \\
\hline \multicolumn{5}{|l|}{ Age group (years) } \\
\hline $15-19$ & $12(75.0 \%)$ & $4(25.0 \%)$ & $0.835(0.245-2.848) \oplus$ & $1.107(0.288-4.246)$ \\
\hline $20-24$ & $59(85.5 \%)$ & $10(14.5 \%)$ & $1.643(0.724-3.731) \oplus$ & $1.785(0.747-4.267)$ \\
\hline $25-29$ & $79(78.2 \%)$ & $22(21.8 \%)$ & 1 & 1 \\
\hline $30-34$ & $24(68.6 \%)$ & $11(31.4 \%)$ & $0.608(0.258-1.430) \oplus$ & $0.453(0.175-1.173)$ \\
\hline $35-39$ & $10(90.9 \%)$ & $1(9.1 \%)$ & $2.785(0.338-22.952) \oplus$ & $2.516(0.286-22.101)$ \\
\hline \multicolumn{5}{|l|}{ Residence } \\
\hline Urban & $169(79.0 \%)$ & $45(21.0 \%)$ & 1 & \\
\hline Rural & $15(83.3 \%)$ & $3(16.7 \%)$ & $1.331(0.369-4.800)$ & \\
\hline \multicolumn{5}{|l|}{ Educational status } \\
\hline $\begin{array}{l}\text { Unable to read and } \\
\text { write }\end{array}$ & $34(72.3 \%)$ & $13(27.7 \%)$ & $0.717(0.311-1.652)$ & \\
\hline Primary & $62(78.5 \%)$ & $17(21.5 \%)$ & 1 & \\
\hline Secondary & $59(83.1 \%)$ & $12(16.9 \%)$ & $1.348(0.593-3.062)$ & \\
\hline Tertiary & $29(82.9 \%)$ & $6(17.1 \%)$ & $1.325(0.473-3.712)$ & \\
\hline \multicolumn{5}{|l|}{ Trimesters } \\
\hline First (<14weeks) & $32(82.1 \%)$ & $7(17.9 \%)$ & $1.213(0.481-3.059)$ & \\
\hline Second (14-28weeks) & $98(79.0 \%)$ & $26(21.0 \%)$ & 1 & \\
\hline Third (>28weeks) & $54(78.3 \%)$ & $15(21.7 \%)$ & $0.955(0.466-1.956)$ & \\
\hline \multicolumn{5}{|l|}{ Gravidity } \\
\hline Primigravidae & $67(78.8 \%)$ & $18(21.2 \%)$ & $0.954(0.495-1.841)$ & \\
\hline Multigravidae & $117(79.6 \%)$ & $30(20.4 \%)$ & 1 & \\
\hline \multicolumn{5}{|l|}{ Habit of eating raw meat } \\
\hline no & $51(64.6 \%)$ & $28(35.4 \%)$ & 1 & 1 \\
\hline yes & $133(86.9 \%)$ & $20(13.1 \%)$ & $3.651(1.890-7.053) \oplus$ & $3.211(1.592-6.477)$ \\
\hline \multicolumn{5}{|c|}{ Habit of eating raw vegetable or fruits } \\
\hline no & $37(66.1 \%)$ & $19(33.9 \%)$ & 1 & 1 \\
\hline yes & $147(83.5 \%)$ & $29(16.5 \%)$ & $2.603(1.317-5.146) \oplus$ & $2.669(1.264-5.639)$ \\
\hline \multicolumn{5}{|c|}{ Presence of domestic animal(s) at home } \\
\hline no & $129(75.9 \%)$ & $41(24.1 \%)$ & 1 & 1 \\
\hline yes & $55(88.7 \%)$ & $7(11.3 \%)$ & $2.497(1.055-5.910) \oplus$ & $3.06(0.827-11.325)$ \\
\hline \multicolumn{5}{|c|}{ Presence of cat(s) at homes } \\
\hline no & $155(77.9 \%)$ & $44(22.1 \%)$ & 1 & 1 \\
\hline yes & $29(87.9 \%)$ & $4(12.1 \%)$ & $2.058(0.687-6.168) \oplus$ & $0.778(0.145-4.171)$ \\
\hline
\end{tabular}




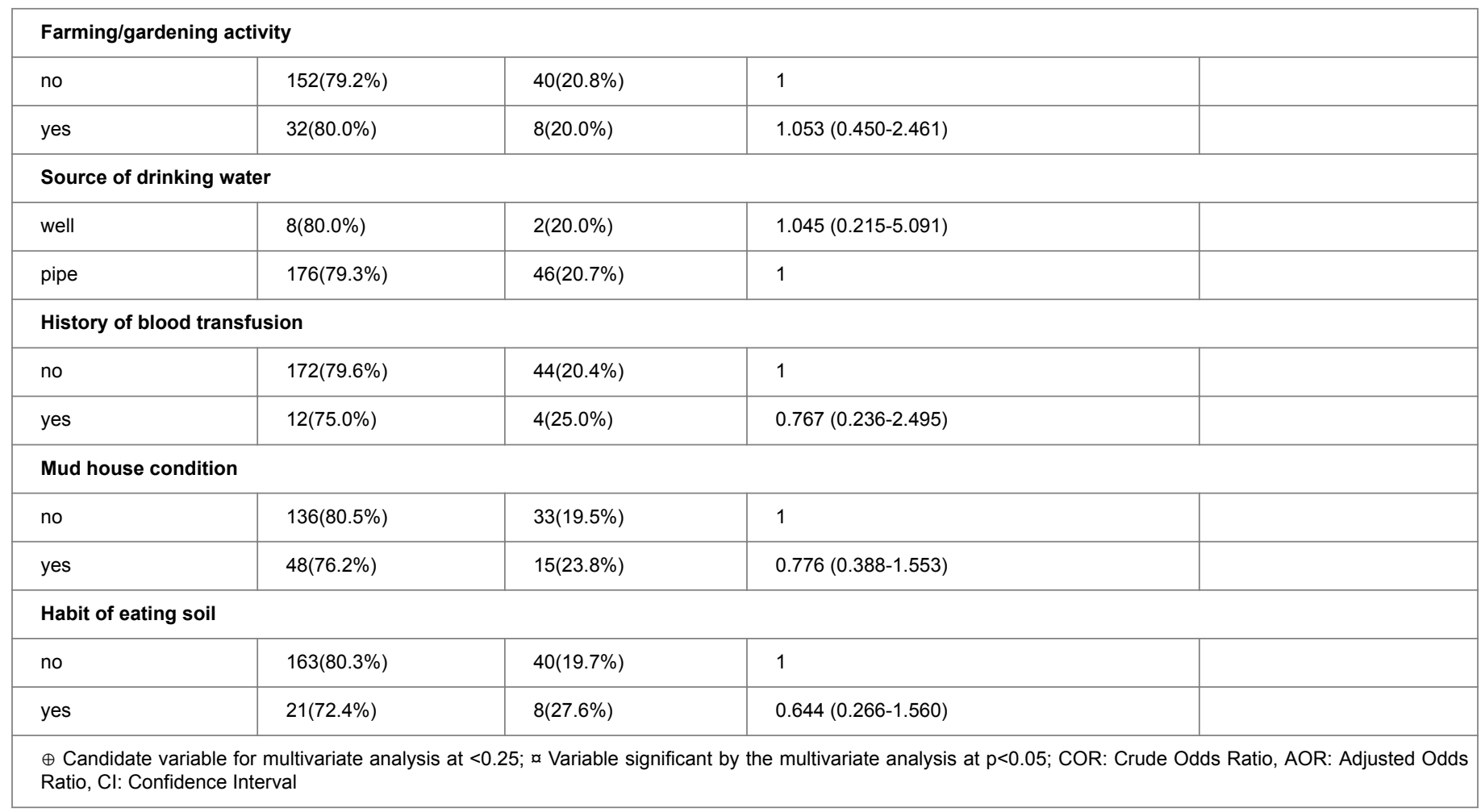

Seropositivity rate with respect to the presence of cats at home, 33 (14.2\%) reported cat's presence, of which $87.9 \%$ $(n=29)$ were found to be seropositive. In the present study 16 (6.9\%) pregnant women had history of blood transfusion, of which 12 (75\%) were seropositive. Forty (17.2\%) of the study participants had reported to have a history of engagement in farming/gardening activities, which could indicate contact with soil and 32 (80\%) of them were seropositive (Table 2 ).

\section{Factors associated with T. gondii infection}

Logistic regression methods were used to identify the main predictor variables associated with $T$. gondii infection. Variables that were entered into multivariate analysis were age, presence of pet animals at home, presence of cats at their home, habit of eating raw meat, habit of eating raw/ unwashed vegetables and fruits. Further analysis using multivariate analysis only two variables, habit of eating raw meat (AOR=3.211; 95\% Cl: 1.592-6.477) and habit of eating raw vegetables or fruits (AOR=2.669; 95\% Cl: 1.264-5.639) were found to be significantly associated with $T$. gondii seropositivity among pregnant women attending ANC in Arba Minch hospital (Table 2).

\section{Discussion}

In the current study, the overall seroprevalence of $T$. gondii infection among pregnant women was $79.3 \%(95 \% \mathrm{Cl}$; 73.7-84.5). One hundred seventy five (75.43\%) were only IgG seropositive, 9 (3.9\%) were IgM sero-positive and two of the nine pregnant women were positive for both IgG and IgM. Overall 9 (3.9\%) pregnant women were positive for IgM antibodies. Presence of IgM antibodies during pregnancy shows the presence of acute $T$. gondii infection and an indication of higher risk of maternal-fetal transmission [22]. Previous study in this regard indicated that in the absence of treatment the risk of congenital infection from acute $T$. gondii infection during pregnancy is about 50\% [23]. Early diagnosis of infections in pregnant mothers is of great importance for early initiation of measures that reduces the risk of transmission and possible sequels on the newborn. Therefore, screening of Toxoplasma infection should be considered as a part of the antenatal investigation during ANC follow up.

The overall seroprevalence of $T$. gondii infection among pregnant women was consistent with studies carried out in Jimma (83.6\%) [17], Central Ethiopia (81.4\%) [18] and Congo (80.3\%) [24]. In contrast to our study, higher seroprevalence was reported among pregnant women in Addis Ababa [25], Gondar (88.6\%) [26], Accra (92.5\%) [14], and in the same study area among HIV infected individuals [19]. On the other hand, lower seroprevalence was reported from Nigeria (40.2\%) [27], Tobago (39.3\%) [28], Tanzania (30.9\%) [29] and Debre Tabor (68.4\%) [30]. The variation in seroprevalence of $T$. gondii found might be due to differences in geographical distribution of the parasite, socio-economic, personal hygienic practices, feeding habit of the study participants and deference in test methods may also account for the variation.

There are inconsistent reports on the association between consumption of raw meat with $T$. gondii infection. In the current study, consumption of raw meat was found to be significantly associated with $T$. gondii seropositivity (AOR=3.211; 95\% Cl: 1.592-6.477). The rate of T. gondii infection was significantly higher and about three times more likely to occur in those eating raw meat compared to those who did not. This finding is in agreement with previous studies 
reported from Northwest Ethiopia [31], Khartoum [32] and Egypt [33]. In contrast, some studies reported absence of association between $T$. gondii infection and consumption of raw meat $[17,34,35]$. The observed differences might be due to the types of meat consumed and the rate of infection in the animals.

The other predictor of $T$. gondii seropositivity in this study is consumption of raw vegetables or fruits, significant association was observed between $T$. gondii seropositivity and eating raw vegetables or fruits. This finding is in agreement with study done in Central Ethiopia [18] and Nigeria [27]. In contrast, studies surveys done in pregnant women in South china [36], Addis Ababa [25] and Felege Hiwot Hospital [31] reported no significant association between the habit of eating raw/ unwashed vegetables and fruits and $T$. gondii infection. The observed differences might be due to differences in feeding habit and hygienic practices of the studied population.

Cats excrete millions of oocysts within a short period of time and play a major role in transmitting $T$. gondii. However, the current study showed that the presence of cats at home was not significantly associated with $T$. gondii seropositivity (Table 2). Similar findings were observed in Saudi Arabia [34], Tobago [28] and Turkey [35]. On the other hand, others documented significant association of cat ownership with $T$. gondii infection $[17,30,36]$. Differences in the types of cats as well as infection rates in cats may account for the observed differences.

In the current study $16(6.5 \%)$ of the study participants responded previous history of blood transfusion and $75 \%$ of whom were T. gondii seropositive. study indicated that blood transfusion is one means of transmission of $T$. gondii infection but, in our study, there was no significant difference in $T$. gondii seropositivity between those who had previous history of blood transfusion and those not having. This finding is in agreement with previous reports $[37,25]$. It is known that only blood donors with acute infection with circulating $T$. gondii parasite in the blood transmit the infection. Since the prevalence of acute infection in blood donors is often very low [38] and [39] received blood is less likely to have tachyzoites stage of the parasite, infective form of the parasite by blood transfusion.

In this study, seroprevalence was found to be higher in age groups [35-38] and T. gondii seropositivity was not significantly differed by age (Table 2 ). This is an agreement with previous studies [25,26]. In contrast, studies in Jimma [17], Turkey [35] and Burkina Faso [40] documented significance difference by age. In summary, all socio-demographic and obstetric (trimester, gravidity) factors assessed none were significantly associated with $T$. gondii seropositivity [41].

\section{Conclusion}

Similar to most studies the overall seroprevalence of $T$. gondii infection was high among pregnant women and serological evidence of primary infection was observed. The high prevalence and presences of primary infection in pregnant women alert the requirement and the need of routine screening of the infection. Therefore, screening of
Toxoplasma infection should be considered during ANC follow up. Consumption of raw meat and raw vegetable or fruits was the main predictors of acquiring $T$. gondii infection among the study participants. Therefore, Health information should be provided to all pregnant women and women who are considering become pregnant on the risk factors predisposing to Toxoplasma infection. Moreover, further studies are recommended to determine incidence of Congenital Toxoplasmosis in the study area.

\section{Acknowledgment}

We would like to thank staff members of Arba Minch Hospital ANC clinic for their cooperation during data collection. We are grateful to Arba Minch Blood Bank center staff for their cooperation during laboratory work. We are also grateful to the study participants.

\section{References}

1. Dubey JP (2010) Toxoplasmosis of Animals and Humans. (2ndedn). Beltseville: CRC Press.

2. Romero OB, Oliveira DM, Neto VFA (2012) Toxoplasmosis: Advances and Vaccine Perspectives. In: Morales AR (Ed). Current Topics in Tropical Medicine. Croatia: InTech, pp: 169-183.

3. Dubey JP, Lindsay DS, Speer CA (1998) Structures of Toxoplasma gondii tachyzoites, bradyzoites, and sporozoites and biology and development of tissue cysts. Clin Microbiol Rev 11: 267-299.

4. Jones JL, Lopez A, Wilson M, Schulkin J, Gibbs R (2001) Congenital Toxoplasmosis: A Review. Obstet Gynecol Surv 56: 296-305.

5. Tenter AM, Heckeroth AR, Weiss LM (2000) Toxoplasma gondii from animals to humans. Int J Parasitol 30: 1217-1258.

6. Montoya JG, Liesenfeld O (2004) Toxoplasmosis. Lancet 363: 1965-1976.

7. Kieffer F, Wallon M (2013) Congenital toxoplasmosis. Handb Clin Neurol 112: 1099-1101.

8. Chaudhry SA, Gad N, Koren G (2014) Toxoplasmosis and pregnancy. Can Fam Physician 60: 334-336.

9. Uttah E, Ogban E, Okonofua C (2013) Toxoplasmosis: A global infection, so widespread, so neglected. Int J Sci Res 3: 1-6.

10. Montoya JG, Remington JS (2002) Management of Toxoplasma gondii infection during pregnancy. Clin Infect Dis 47: 554-566.

11. Montoya JG (2002) Laboratory diagnosis of Toxoplasma gondii infection and Toxoplasmosis. J Infec Dis 185: 73-82.

12. Sakikawa $M$, Noda $S$, Hanaoka $M$, Nakayama $H$, Hojo $S$, et al. (2012) Anti-toxoplasma antibody prevalence, primary infection rate, and risk factors in a study of Toxoplasmosis in 4,466 pregnant women in Japan. Clin Vaccine Immunol 19: 365-367.

13. Sroka S, Bartelheimer N, Winter A, Heukelbach J, Ariza L, et al. (2010) Prevalence and risk factors of toxoplasmosis among pregnant women in Fortaleza, Northeastern Brazil. Am J Trop Med Hyg 83: 528-533.

14. Ayi I, Edu AAS, Apea-Kubi KA, Boamah D, Bosompem KM, et al. (2009) Sero-epidemiology of toxoplasmosis amongst pregnant women in the greater accra region of Ghana. Ghana Med J 43: 107-114. 
15. Alsammani MA (2014) Sero-epidemiology and risk factors for Toxoplasma gondii among pregnant women in Arab and African countries. J Parasitic Dis 40: 569-579.

16. Gebremedhin EZ, Tadesse G (2015) A meta-analysis of the prevalence of Toxoplasma gondii in animals and humans in Ethiopia. Parasites Vectors 8: 291.

17. Zemene E, Yewhalaw D, Abera S, Belay T, Samuel A, et al. (2012) Seroprevalence of Toxoplasma gondii and associated risk factors among pregnant women in Jimma town, Southwestern Ethiopia. BMC Infect Dis 12: 337

18. Gebremedhin EZ, Abebe AH, Tessema TS, Tullu KD, Medhin G, et al. (2013) Seroepidemiology of Toxoplasma gondii infection in women of child-bearing age in central Ethiopia. BMC Infect Dis 13: 101.

19. Yohanes T, Debalke S, Zemene E (2014) Latent Toxoplasma gondii infection and associated risk factors among HIV-infected individuals at Arba Minch Hospital, South Ethiopia. AIDS Research and Treatment.

20. Dawit G, Shishay K (2014) Epidemiology, public health impact, and control methods of the most neglected parasite diseases in ethiopia: a review. World J Med Sci 10: 94-102.

21. Semaw H, Awet M, Yohannes $T$ (2015) Sero-prevalence of Hepatitis B surface antigen and associated factors among pregnant mothers attending antenatal care service, mekelle, ethiopia: evidence from institutional based quantitative crosssectional study, world academy of science, engineering and technology medical and health sciences 2 .

22. Tekkesin N (2012) Diagnosis of toxoplasmosis in pregnancy: $A$ review. HOAJ Biology.

23. Paquet $\mathrm{C}$, Yudin $\mathrm{MH}$, Allen VM, Bouchard $\mathrm{C}$, Boucher $\mathrm{M}$, et al. (2013) Toxoplasmosis in pregnancy: Prevention, screening, and treatment. J Obstet Gynaecol Can 35: 78-81.

24. Doudou $Y$, Renaud $P$, Coralie L, Jacqueline F, Hypolite $S$, et al. (2014) Toxoplasmosis among pregnant women: High seroprevalence and risk factors in Kinshasa, Democratic Republic of Congo. Asian Pac J Trop Biomed 4: 69-74.

25. Gelaye W, Kebede T, Hailu A (2015) High prevalence of antitoxoplasma antibodies and absence of Toxoplasma gondii infection risk factors among pregnant women attending routine antenatal care in two Hospitals of Addis Ababa, Ethiopia. Int J Infect Dis 34: 41-45.

26. Endris M, Belyhun $Y$, Moges F, Adefiris M, Tekeste Z, et al. (2014) Seroprevalence and associated risk factors of toxoplasma gondii in pregnant women attending in Northwest Ethiopia. Iranian J Parasitol 9: 407-414.

27. Agboola AM, Busari OS, Osinupebi OA, Amoo AOJ (2011) Seroprevalence of Toxoplasma gondii antibodies among pregnant women attending antenatal clinic of federal medical center, Lagos, Nigeria. Int J Biol Med Res 2: 1135-1139.

28. Ramsewak S, Gooding R, Ganta K, Seepersadsingh N, Adesiyun AA (2008) Seroprevalence and risk factors of Toxoplasma gondii infection among pregnant women in Trinidad and Tobago. Rev Panam Salud Publica 23: 164-170.

29. Mwambe B, Mshana SE, Kidenya BR, Massinde AN, Mazigo HD, et al. (2013) Sero-prevalence and factors associated with Toxoplasma gondii infection among pregnant women attending antenatal care in Mwanza, Tanzania. Parasites Vector 6: 222.

30. Agmas B, Tesfaye R, Koye DN (2015) Seroprevalence of Toxoplasma gondii infection and associated risk factors among pregnant women in Debre Tabor, Northwest Ethiopia. BMC Res Notes 8: 107.

31. Awoke K, Nibret E, Munshea A (2015) Sero-prevalence, and associated risk factors of Toxoplasma gondii infection among pregnant women attending antenatal care at Felege Hiwot Referral Hospital, northwest Ethiopia. Asian Pacific J Trop Med 8: 549-554.

32. Khalil KM, Ahmed AA, Elrayah E (2012) Seroprevalence of Toxoplasma gondii Infection in Humans in Khartoum State, Sudan. Int J Trop Med 7: 143-150.

33. Kamal AM, Ahmed AK, Abdellatif MZM, Tawfik M, Hassan EE (2015) Seropositivity of Toxoplasmosis in pregnant women by ELISA at Minia University Hospital, Egypt. Korean J Parasitol 53: 605-610.

34. Al-Harthi SA, Jamjoom MB, Ghazi HO (2006) Seroprevalence of Toxoplasma gondii among pregnant women in Makkah, Saudi Arabia Umm Al-Qura University. J Sci Med Eng 18: 217-227.

35. Ertug S, Okyay P, Turkmen M, Yuksel H (2005) Seroprevalence and risk factors for toxoplasma infection among pregnant women in Aydin province, Turkey. BMC Pub Health 5: 66.

36. Nissapatorn V, Suwanrath C, Sawangjaroen N, Ling LY, Chandeying V (2011) Toxoplasmosis-serological evidence, and associated risk factors among pregnant women in Southern Thailand. Am J Trop Med Hyg 85: 243-247.

37. Esquivel CA, Álvarez AS, Duarte SGN, Martínez SE, García JHD, et al. (2006) Seroepidemiology of Toxoplasma gondii infection in pregnant women in a public hospital in northern Mexico. BMC Infect Dis 6: 113.

38. Walle F, Kebede N, Tsegaye A, Kassa T (2013) Seroprevalence and risk factors for Toxoplasmosis in HIV infected and noninfected individuals in Bahir Dar, Northwest Ethiopia. Parasites Vector 6: 15 .

39. Modrek MJ, Mousavi M, Saravani R (2014) Toxoplasma gondii seroprevalence among blood donors in Zahedan, Southeastern Iran. Int J Infec 1: e21111.

40. Simpore J, Savadogo A, Ilboudo D, Nadambega MC, Esposito M, et al. (2006) Toxoplasma gondii, HCV, and HBV seroprevalence and co-infection among HIV-positive and negative pregnant women in Burkina Faso. J Med Virol 78: 730-733.

41. Duan C, Ning Z, Hao W, Luo X, Tan J, et al. (2012) Toxoplasma gondii infection among pregnant women in Guangdong province, Subtropical Southern China. J Med Microb Diagn 1:3. 